\title{
A Note on $n$-Set Distance-Labelings of Graphs
}

\author{
Roger K. Yeh \\ Department of Applied Mathematics, Feng Chia University, Taiwan \\ Email: rkyeh@fcu.edu.tw
}

How to cite this paper: Yeh, R.K. (2021) A Note on $n$-Set Distance-Labelings of Graphs. Open Journal of Discrete Mathematics, 11, 55-60.

https://doi.org/10.4236/ojdm.2021.113005

Received: March 2, 2021

Accepted: June 5, 2021

Published: June 8, 2021

Copyright (c) 2021 by author(s) and Scientific Research Publishing Inc. This work is licensed under the Creative Commons Attribution International License (CC BY 4.0).

http://creativecommons.org/licenses/by/4.0/

\begin{abstract}
This note is considered as a sequel of Yeh [1]. Here, we present a generalized (vertex) distance labeling (labeling vertices under constraints depending the on distance between vertices) of a graph. Instead of assigning a number (label) to each vertex, we assign a set of numbers to each vertex under given conditions. Some basic results are given in the first part of the note. Then we study a particular class of this type of labelings on several classes of graphs.
\end{abstract}

\section{Keywords \\ Graph Distance Labeling}

\section{Introduction}

Inspired by a channel assignment problem proposed by Roberts [2] in 1988, Griggs and Yeh [3] formulated the $L(2,1)$-labeling problem for graphs. There are considerable amounts of articles studying this labeling and its generalizations or related problems. Readers can see [4] or [5] for a survey. In this note, we like to consider a generalization of the $L(2,1)$-labeling. Let $A$ and $B$ be two subsets of natural numbers. Define $\|A-B\|=\min \{|a-b|: a \in A, b \in B\}$. Denote the set $[k]=\{0,1, \cdots, k\}$ and $\left(\begin{array}{c}{[k]} \\ n\end{array}\right)$ the collection of all $n$-subsets of $[k]$.

Motivated by the article [6], we propose the following labeling on a graph.

Let $G=(V, E)$ be a graph and $n$ be a positive integer. Given non-negative integers $\delta_{1} \geq \delta_{2}$ an $L^{(n)}\left(\delta_{1}, \delta_{2}\right)$-labeling is a function $f: V(G) \rightarrow\left(\begin{array}{c}{[k]} \\ n\end{array}\right)$ for some $k \geq 1$ such that $|f(u)-f(v)| \geq \delta_{i}$ whenever the distance between $u$ and $v$ in $G$ is $i$, for $i=1,2$. (The minimum value and the maximum value of $\bigcup_{v \in V(G)} f(v)$ is 0 and $k$, respectively.) The value $k$ is called the span of $f$. The smallest $k$ so that there is an $L^{(n)}\left(\delta_{1}, \delta_{2}\right)$-labeling $f$ with span $k$, is denoted by $\lambda^{(n)}\left(G ; \delta_{1}, \delta_{2}\right)$ and called the $L^{(n)}\left(\delta_{1}, \delta_{2}\right)$-labeling number of $G$. An $L^{(n)}\left(\delta_{1}, \delta_{2}\right)$ 
-labeling with span $\lambda^{(n)}\left(G ; \delta_{1}, \delta_{2}\right)$ is called an optimal $L^{(n)}\left(\delta_{1}, \delta_{2}\right)$-labeling. If $n=1$ then notations $L^{(1)}$ and $\lambda^{(1)}$ will be simplified as $L$ and $\lambda$, respectively.

Note: 1) The elements in $[k]$ are called "numbers" and $f(u)$ is called the "label" of $u$. So, a label is a set in this problem. 2) Using our notation, the labeling in [6] is the $L\left(\delta_{1}, 0\right)$-labeling for $\delta_{1} \geq 1$.

Previously, we have studied the $L^{(2)}(2,1)$-labeling problem (cf. [1]). In this note, we will first investigate properties of the $L^{(n)}\left(\delta_{1}, \delta_{2}\right)$ for $n \geq 1$. Then, we study the case of $\left(\delta_{1}, \delta_{2}\right)=(1,1)$.

\section{Preliminarily}

Let $G$ be a graph and $n$ an positive integer. Now, we construct a new graph $G^{(n)}$ by replacing each vertex $v$ in $G$ by $n$ vertices $v_{i}, 1 \leq i \leq n$ and $u_{i}$ is adjacent to $v_{j}$ for all $i, j$, in $G^{(n)}$, whenever $u$ and $v$ is adjacent in $G$. That is, $u_{i}$ and $v_{j}$, for all $i, j$, induces a complete bipartite graph $K_{n, n}$. Note that $G^{(1)}=G$.

It is easy to verify that $\lambda^{(n)}\left(G ; \delta_{1}, 1\right)=\lambda\left(G^{(n)} ; \delta_{1}, 1\right)$. Thus, for example, $\lambda^{(n)}\left(K_{m} ; 2,1\right)=\lambda\left(K_{n, n, \cdots, n} ; 2,1\right)=n m+m-2$, where $m \geq 2$, by previous result on complete $m$-partite graph $K_{n, n, \cdots, n}$ (cf. [3]).

Next, we consider the relation between the labeling numbers for $n=1$ and $n \geq 1$. In the following, $\lambda(G ; 1,1)$ and $\lambda^{(n)}(G ; 1,1)$ are denoted by $\lambda_{1}(G)$ and $\lambda_{1}^{(n)}(G)$, respectively, for short.

Proposition 2.1. Let $n \geq 1, \delta_{1} \geq \delta_{2}$ be nonnegative integers and $\Delta$ be the maximum degree of $G$. Then

1) $(n-1)(\Delta+1)+\delta_{1}+(\Delta-1) \delta_{2} \leq \lambda^{(n)}\left(G ; \delta_{1}, \delta_{1}\right)$.

2) $\lambda^{(n)}\left(G ; \delta_{1}, \delta_{1}\right) \leq \lambda\left(G ; n+\delta_{1}-1, n+\delta_{2}-1\right)+n-1$.

\section{Proof.}

1) A vertex $u$ with the maximum degree $\Delta$ in a graph $G$ is called a major vertex of $G$. By counting the numbers for the labels of a major vertex and its neighbors and numbers need to separate each label (the $\left(\delta_{1}, \delta_{2}\right)$ condition), we shall have the trivial lower bound.

2) Let $\lambda\left(G ; n+\delta_{1}-1, n+\delta_{2}-1\right)=k$ and $f$ an optimal $L\left(n+\delta_{1}-1, n+\delta_{2}-1\right)$ -labeling. Define sets $L_{i}=\{i, i+1, \cdots, i+n-1\}, i=0,1, \cdots, k$ and function $g_{f}: V(G) \rightarrow\left(\begin{array}{c}{[k+n-1]} \\ n\end{array}\right)$ by $g_{f}(u)=L_{i}$ whenever $f(u)=i$ for $i=0,1, \cdots, k$.

Let $u$ and $v$ be distinct vertices with $d_{G}(u, v)=j$ for $j=1,2$ in $G$. Suppose $f(u)=i$ and $f(v)=i+n+\delta_{j}^{\prime}-1$ for $\delta_{j}^{\prime} \geq \delta_{j}$ for $j=1,2$. Then $g_{f}(u)=\{i, i+1, \cdots, i+n-1\}$ and $g_{f}(v)=\left\{i+n+\delta_{j}^{\prime}-1, i+n+\delta_{j}^{\prime}, \cdots, i+n+\delta_{j}^{\prime}-1+n-1\right\}$. Hence $\left\|g_{f}(u)-g_{f}(v)\right\|=\left(i+n+\delta_{j}^{\prime}\right)-(i+n-1)=\delta_{j}^{\prime} \geq \delta_{j}$ for $j=1,2$. Thus $g_{f}$ is an $L^{(n)}\left(\delta_{1}, \delta_{2}\right)$-labeling with span $k+n-1$. Therefore $\lambda^{(n)}\left(G ; \delta_{1}, \delta_{1}\right) \leq \lambda\left(G ; n+\delta_{1}-1, n+\delta_{2}-1\right)+n-1$.

The following is the direct consequence of Proposition 2.1 when $\left(\delta_{1}, \delta_{1}\right)=(1,1)$. 
Also notice that $\lambda\left(G ; d \delta_{1}, d \delta_{2}\right)=d \lambda\left(G ; \delta_{1}, \delta_{2}\right)$.

Corollary 2.2. Let $\Delta$ be the maximum degree of $\mathrm{G}$. Then

$$
(\Delta+1) n-1 \leq \lambda_{1}^{(n)}(G) \leq n \lambda_{1}(G)+n-1 .
$$

By Corollary 2.2, we know that whenever $\lambda_{1}(G)=\Delta$, the lower bound and the upper bound are equal and hence $\lambda_{1}^{(n)}(G)=(\Delta+1) n-1$. There are several well-known classes of graphs whose $\lambda_{1}$ values are all $\Delta$ (see [7]). For example, tree $T$, wheel $W_{m}$ (with $m$ rims), the square lattice $\Gamma_{S}$ (4-regular infinite plane graph), the hexagonal lattice $\Gamma_{H}$ (3-regular infinite plane graph), and the triangular lattice $\Gamma_{\Delta}$ (6-regular infinite plane graph) are all with $\lambda_{1}=\Delta$. We summarize as follows.

\section{Theorem 2.3.}
1) $\lambda_{1}^{(n)}(T)=(\Delta(T)+1) n-1$.
2) $\lambda_{1}^{(n)}\left(W_{m}\right)=(m+1) n-1$.
3) $\lambda_{1}^{(n)}\left(\Gamma_{S}\right)=5 n-1$.
4) $\lambda_{1}^{(n)}\left(\Gamma_{H}\right)=4 n-1$.
5) $\lambda_{1}^{(n)}\left(\Gamma_{\Delta}\right)=7 n-1$.

\section{Cycles}

We know that the maximum degree of a cycle $C_{m}$ of order $m \geq 3$ is 2 . However, $\lambda_{1}\left(C_{m}\right)$ is not necessary 2 . It depends on $m$. In this section, we will consider $L^{(n)}(1,1)$-labelings on cycles.

Proposition 3.1. Let $C_{m}$ be a cycle of order $m \geq 3$. Then $\lambda_{1}^{(n)}\left(C_{m}\right)=3 n-1$ if $m \equiv 0(\bmod 3)$.

Proof. Since the maximum degree of $C_{m}$ is 2 , the trivial lower bound is $3 n-1$ by Corollary 2.2. On the other hand, we use $\{0,1, \cdots, n-1\},\{n, n+1, \cdots, 2 n-1\}$ and $\{2 n, 2 n+1, \cdots, 3 n-1\}$ consecutively to label vertices of $C_{m}$ where $m \equiv 0(\bmod 3)$, to obtain an $L^{(n)}(1,1)$-labeling of $C_{m}$ with span $3 n-1$. Thus, we have the exact value of $\lambda_{1}^{(n)}\left(C_{m}\right)$ in this case.

Lemma 3.2. Let $C_{m}$ be a cycle of order $m$ where $m \neq 0(\bmod 3)$. Then $\lambda_{1}^{(n)}\left(C_{m}\right) \geq 3 n$.

Proof. Let $V\left(C_{m}\right)=\left\{v_{1}, v_{2}, \cdots, v_{m}\right\}$ where $v_{i}$ is adjacent to $v_{i+1}$ for $i=1,2, \cdots, m$ where $v_{m+1}=v_{1}$. Suppose $\lambda_{1}^{(n)}\left(C_{m}\right) \leq 3 n-1$. Let $f$ be an $L^{(n)}(1,1)$ -labeling with span $3 n-1$. Let $f\left(v_{1}\right)=A, f\left(v_{2}\right)=B$ and $f\left(v_{3}\right)=C$. Since, by definition, $f\left(v_{1}\right), f\left(v_{2}\right)$ and $f\left(v_{3}\right)$ are distinct, that is, $|A \cup B \cup C|=3 n$ and $A \cup B \cup C=[3 n-1]$. Now, $f\left(v_{4}\right) \cap(B \cup C)=\varnothing$ and $f\left(v_{4}\right) \subseteq[3 n-1]$. Hence $f\left(v_{4}\right)=A$. Consider $f\left(v_{5}\right)$. Again, we have $f\left(v_{5}\right) \cap(A \cup C)=\varnothing$ and $f\left(v_{5}\right) \subseteq[3 n-1]$. Hence $f\left(v_{5}\right)=B$. In general, we have 1) $f\left(v_{i}\right)=A$ if $i \equiv 1(\bmod 3), 2) \quad f\left(v_{i}\right)=B$ if $i \equiv 2(\bmod 3)$ and 3$) \quad f\left(v_{i}\right)=C$ if $i \equiv 0(\bmod 3)$, for $i=1,2, \cdots, m$.

If $m \equiv 1(\bmod 3)$ then $f\left(v_{m}\right)=A$. But $v_{m}$ is adjacent to $v_{1}$, where $f\left(v_{1}\right)=A$. This violates the condition on adjacent vertices. If $m \equiv 2(\bmod 3)$ then $f\left(v_{m}\right)=B=f\left(v_{2}\right)$ while the distance between $v_{m}$ and $v_{2}$ is 2. Again, 
this violates the condition on distance 2 vertices. We have a contradiction on each case. Therefore, $\lambda_{1}^{(n)}\left(C_{m}\right) \geq 3 n$ for $m \neq \equiv 0(\bmod 3)$.

Proposition 3.3. If 1$) m \equiv 1(\bmod 3)$ and $m \geq 3 n+1$ or 2$) m \equiv 2(\bmod 3)$ and $m \geq 6 n+2$ then $\lambda_{1}^{(n)}\left(C_{m}\right)=3 n$.

Proof. Let $V\left(C_{m}\right)=\left\{v_{1}, v_{2}, \cdots, v_{m}\right\}$.

1) Suppose $m=3 n+1$ and Define $A_{0}=\{0,1, \cdots, n-1\}$, $A_{1}=\{n, n+1, \cdots, 2 n-1\}, \quad A_{2}=\{2 n, 2 n+1, \cdots, 3 n-1\} \quad$ and $\quad A_{3}=\{3 n, 0, \cdots, n-2\}$. Denote $X-i(\bmod k)$ to be that set $\{x-i(\bmod k): x \in X\}$. Then we use $A_{1}, A_{2}, A_{3}, A_{1}-1, A_{2}-1, A_{3}-1$, $A_{1}-2, A_{2}-2, A_{3}-2, \cdots, A_{1}-(n-1), A_{2}-(n-1), A_{3}-(n-1)$ to label $v_{1}, v_{2}, \cdots, v_{3 n}$. The last vertex $v_{3 n+1}$ is labeled by $A_{0}$. We see that this is an $L^{(n)}(1,1)$-labeling with span $3 n$ of $C_{m}$.

Suppose $m>3 n+1$. Then we label first $3 n+1$ vertices as we did above. And then we repeatedly use $A_{0}, A_{1}$ and $A_{2}$ to label remaining vertices.

2) First consider $m=6 n+2$. We use the sequence presented in (1) for $m=3 n+1$ twice to label vertices of $C_{6 n+2}$. Obviously, it is still an $L^{(n)}(1,1)$ -labeling for $C_{6 n+2}$ with span $3 n$.

For $m>6 n+2$, we label the first $6 n+1$ vertices (namely, $v_{1}, v_{2}, \cdots, v_{6 n+1}$ ) using the same sequence as above and then repeat using $A_{0}, A_{1}$ and $A_{2}$ to label remaining vertices. Thus $\lambda_{1}^{(n)}\left(C_{m}\right) \leq 3 n$ in each case. On the other hand, by Lemma 3.2, we have the equality.

Lemma 3.4. Let $G$ be a diameter two graph with order $p$. Then $\lambda_{1}^{(n)}(G)=n p-1$.

Proof. Since $G$ is a diameter two graph, every vertex must receive distinct label. Thus, we need at least $n p$ numbers, i.e., $\lambda_{1}^{(n)}(G)=n p-1$. On the other hand, we can use $\{$ in, in $+1, \cdots, i n+n-1\}$ for $i=0,1, \cdots, p-1$ to label vertices of $G$ in any order. Hence $\lambda_{1}^{(n)}(G) \leq n p-1$.

Corollary 3.5 .

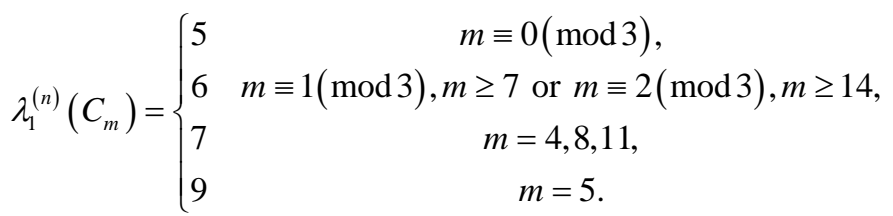

Proof. Let $V\left(C_{m}\right)=\left\{v_{1}, v_{2}, \cdots, v_{m}\right\}$ where $v_{i}$ is adjacent to $v_{i+1}$ for $i=1,2, \cdots, m$ where $v_{m+1}=v_{1}$.

Claim 1. $\lambda_{1}^{(2)}\left(C_{8}\right)=7$.

Suppose $\lambda_{1}^{(2)}\left(C_{8}\right) \leq 6$. Let $f$ be an $L^{(2)}(1,1)$-labeling with span 6. Since $m=8$, there must have three consecutive vertices, say $v_{1}, v_{2}$ and $v_{3}$, be labeled without using 6; and let $6 \in f\left(v_{4}\right)$. Also let $f\left(v_{1}\right)=\left\{a_{1}, a_{2}\right\}, f\left(v_{2}\right)=\left\{b_{1}, b_{2}\right\}$ and $f\left(v_{3}\right)=\left\{c_{1}, c_{2}\right\}$. Then $f\left(v_{4}\right)=\{a, 6\}$ where $a=a_{1}$ or $a_{2}$. Suppose $a=a_{1}$. Hence $f\left(v_{5}\right) \subseteq\left\{b_{1}, b_{2}, a_{2}\right\}$ and $f\left(v_{8}\right) \subset\left\{c_{1}, c_{2}, 6\right\}$. Since $\left(f\left(v_{6}\right) \cup f\left(v_{7}\right)\right) \cap\left(f\left(v_{5}\right) \cup f\left(v_{8}\right)\right)=\varnothing$, we left only 3 numbers for $f\left(v_{6}\right) \cup f\left(v_{7}\right)$, (that is two from $\left\{b_{1}, b_{2}, a_{2}, c_{1}, c_{2}, 6\right\} \backslash\left(f\left(v_{5}\right) \cup f\left(v_{8}\right)\right)$ plus $\left.a_{1}\right)$. It is not enough. The case for $a=a_{2}$ is similar. 
Thus, $\lambda_{1}^{(2)}\left(C_{8}\right) \geq 7$. On the other hand, we can use $\{0,1\},\{2,3\},\{4,5\},\{6,7\}$ consecutively to label $v_{1}, v_{2}, \cdots, v_{8}$ to obtain an $L^{(2)}(1,1)$-labeling with sapn 7 . Hence the claim holds.

Claim 2. $\lambda_{1}^{(2)}\left(C_{11}\right)=7$.

Let $f$ be an $L^{(2)}(1,1)$-labeling with span 6. Similar to Claim 1, we may assume that $f\left(v_{1}\right)=\left\{a_{1}, a_{2}\right\}, f\left(v_{2}\right)=\left\{b_{1}, b_{2}\right\}, \quad f\left(v_{3}\right)=\left\{c_{1}, c_{2}\right\} \quad$ and $f\left(v_{4}\right)=\{6, a\}$ where $a \in\left\{a_{1}, a_{2}\right\}$ and $6 \notin\left\{a_{1}, a_{2}, b_{1}, b_{2}, c_{1}, c_{2}\right\}$.

Again, we have $f\left(v_{11}\right) \subset\left\{c_{1}, c_{2}, 6\right\}$. Consider the following cases:

1) $f\left(v_{8}\right) \subset\left\{c_{1}, c_{2}, 6\right\}$. Since $f\left(v_{4}\right)=\{6, a\}$ (as indicated above), the discussion on $f\left(v_{4}\right), f\left(v_{5}\right), f\left(v_{6}\right), f\left(v_{7}\right)$ and $f\left(v_{8}\right)$ is the same as Claim 1.

2) $f\left(v_{8}\right) \subset\left\{a_{1}, a_{2}, b_{1}, b_{2}, 6\right\}$. Since $f\left(v_{1}\right)=\left\{a_{1}, a_{2}\right\}, f\left(v_{10}\right) \cap\left\{a_{1}, a_{2}\right\}=\varnothing$. Let $c \in\left\{c_{1}, c_{2}, 6\right\} \backslash f\left(v_{11}\right)$. Hence $f\left(v_{9}\right) \subset\left\{a_{1}, a_{2}, c\right\}$. So $f\left(v_{8}\right) \subset\left\{b_{1}, b_{2}, c\right\}$. Thus, there is only one number left available for $f\left(v_{10}\right)$. This is a contradiction.

3) Suppose $f\left(v_{8}\right)$ consists of one number of $f\left(v_{1}\right)$ and one number of $f\left(v_{3}\right)$. Without loss of generality, say $f\left(v_{8}\right)=\left\{a_{1}, c_{1}\right\}$. Then $f\left(v_{5}\right) \subset\left\{b_{1}, b_{2}, a^{\prime}\right\}$ where $a^{\prime}=a_{2}$ if $a=a_{1}$ and vice versa. Then there only three numbers available for $f\left(v_{6}\right) \cup f\left(v_{7}\right)$ and they are one from $\left\{b_{1}, b_{2}, a^{\prime}\right\} \backslash f\left(v_{5}\right), c_{1}$ and 6 . That is not enough.

Therefore, $\lambda_{1}^{(2)}\left(C_{11}\right) \geq 7$. On the other hand, we can use $\{0,1\},\{2,3\},\{4,5\},\{6,7\}$ consecutively to label $v_{1}, v_{2}, \cdots, v_{11}$ to obtain an $L^{(2)}(1,1)$-labeling with span 7 . Hence the claim holds. Finally, we have

1) $m \equiv 0(\bmod 3)$.

By Proposition 3.2, $\lambda_{1}^{(2)}\left(C_{m}\right)=5$.

2) $m \equiv 1(\bmod 3)$.

By Proposition 3.3, $\lambda_{1}^{(2)}\left(C_{m}\right)=6$ if $m \geq 7$. Since $C_{4}$ is diameter 2 graph, by Lemma 3.4, $\lambda_{1}^{(2)}\left(C_{4}\right)=7$.

3) $m \equiv 2(\bmod 3)$.

By Proposition 3.3, $\lambda_{1}^{(2)}\left(C_{m}\right)=6$ if $m \geq 14$. Case for $m=11$ and 8 are obtained by Claim 1 and Claim 2. Since $C_{5}$ is also a diameter 2 graph, by Lemma 3.4, $\lambda_{1}^{(2)}\left(C_{5}\right)=9$.

\section{Concluding Remark}

We have obtained values of $\lambda_{1}^{(2)}\left(C_{m}\right)$ for all $m$ and $\lambda_{1}^{(n)}\left(C_{m}\right)$ for some $m$ where $n \geq 3$. Otherwise, the labeling numbers are still unknown. It is known that $\lambda_{1}\left(C_{m}\right)=4$ if $m \neq 0(\bmod 3)$ (cf. [8]). Hence an upper bound is $4 n-1$ in this case. On the other hand, the lower bound we have in Lemma 3.2 is $3 n$. Thus, there is still a gap between $3 n$ and $4 n-1$ for $n>1$.

\section{Acknowledgements}

The author would like to thank the referee for valuable editorial suggestions.

\section{Conflicts of Interest}

The author declares no conflicts of interest regarding the publication of this paper. 


\section{References}

[1] Yeh, R.K. (2019) Pair L(2,1)-Labelings of Infinite Graphs. Discussiones Mathematicae Graph Theory, 39, 257-269. https://doi.org/10.7151/dmgt.2077

[2] Roberts, F.S. (1988) Private Communication with Griggs J. R.

[3] Griggs, J.R. and Yeh, R.K. (1992) Labelling Graphs with a Condition at Distance 2. SIAM Journal on Discrete Mathematics, 5, 586-595. https://doi.org/10.1137/0405048

[4] Calamoneri, T. (2011) The L(h,k)-Labeling Problems: A Update Survey and Annotated Bibliography. The Computer Journal, 54, 1344-1371. https://doi.org/10.1093/comjnl/bxr037

[5] Yeh, R.K. (2006) A Survey on Labeling Graphs with a Condition at Distance Two. Discrete Mathematics, 306, 1217-1231. https://doi.org/10.1016/j.disc.2005.11.029

[6] Füredi, Z., Griggs, J.R. and Kleitman, D.J. (1989) Pair Labellings with Given Distance. SIAM Journal on Discrete Mathematics, 2, 491-499. https://doi.org/10.1137/0402044

[7] Griggs, J.R. and Jin, X.T. (2008) Real Number Channel Assignments for Lattices. SIAM Journal on Discrete Mathematics, 22, 996-1021. https://doi.org/10.1137/060650982

[8] Liu, D.D.-F. and Yeh, R.K. (1997)On Distance Two Labelings of Graphs. Ars Combinatoria, 47, 13-22. 